\title{
Circular polarization of radio emission from air showers in thun- derstorm conditions
}

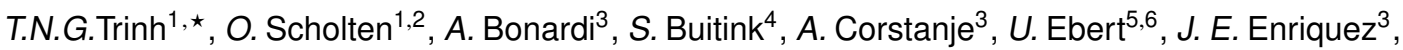

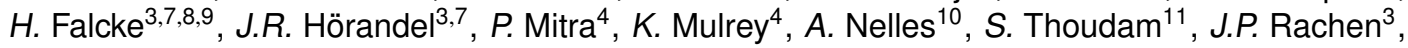 \\ L. Rossetto ${ }^{3}, C$. Rutjes ${ }^{5}, P$. Schellart ${ }^{12}, S$. ter Veen ${ }^{3}$, and T. Winchen ${ }^{4}$ \\ ${ }^{1}$ KVI-Center for Advanced Radiation Technology, University Groningen, P.O. Box 72, 9700 AB Groningen, \\ The Netherlands \\ ${ }^{2}$ Interuniversity Institute for High-Energy, Vrije Universiteit Brussel, Pleinlaan 2, 1050 Brussels, Belgium \\ ${ }^{3}$ Department of Astrophysics/IMAPP, Radboud University Nijmegen, P.O. Box 9010, 6500 GL Nijmegen, The \\ Netherlands \\ ${ }^{4}$ Astrophysical Institute, Vrije Universiteit Brussel, Pleinlaan 2, 1050 Brussels, Belgium \\ ${ }^{5}$ Center for Mathematics and Computer Science (CWI), PO Box 94079, 1090 GB Amsterdam, The Nether- \\ lands \\ ${ }^{6}$ Department of Applied Physics, Eindhoven University of Technology (TU/e), PO Box 513, 5600 MB Eind- \\ hoven, The Netherlands \\ ${ }^{7}$ NIKHEF, Science Park Amsterdam, 1098 XG Amsterdam, The Netherlands \\ ${ }^{8}$ Netherlands Institute of Radio Astronomy (ASTRON), Postbus 2, 7990 AA Dwingeloo, The Netherlands \\ ${ }^{9}$ Max-Planck-Institut für Radioastronomie, P.O. Box 20 24, Bonn, Germany \\ ${ }^{10}$ Department of Physics and Astronomy, University of California Irvine, Irvine, CA 92697-4575, USA \\ ${ }^{11}$ Department of Physics and Electrical Engineering, Linnéuniversitetet, 35195 Växjö, Sweden \\ ${ }^{12}$ Department of Astrophysical Sciences, Princeton University, Princeton, NJ 08544, USA
}

\begin{abstract}
We present measured radio emission from cosmic-ray-induced air showers under thunderstorm conditions. We observe for these events large differences in intensity, linear polarization and circular polarization from the events measured under fair-weather conditions. This can be explained by the effects of atmospheric electric fields in thunderclouds. Therefore, measuring the intensity and polarization of radio emission from cosmic ray extensive air showers during thunderstorm conditions provides a new tool to probe the atmospheric electric fields present in thunderclouds.
\end{abstract}

\section{Introduction}

Lightning initiation [1] and propagation [2] are driven by the electric fields in a thunderstorm. As it is very challenging to measure these fields in situ before or after the discharge and up to now essentially impossible during the discharge, there are ongoing controversies related to the physical mechanisms. A new non-intrusive method of field measurements is offered with LOFAR. It was discovered that under thunderstorm conditions, the intensity and polarizations of radio emission from

^e-mail: t.n.g.trinh@rug.nl 
air showers differ considerably [3] from events in fair-weather conditions, so they can be used to probe the atmospheric electric fields present in thunderclouds.

Radio emission from air showers is measured by many experiments. Here we present the measurement with the LOw-Frequency ARay (LOFAR) radio telescope using the Low-Band Antennas (LBA). The LBA, having the frequency range of $10-90 \mathrm{MHz}$, are grouped into circular stations. The core of LOFAR, so-called 'Superterp', consists of 6 such stations, located in a 320 m diameter region. The read-out of the LOFAR antennas for a cosmic-ray event is triggered by the LORA particle detector array at the Superterp. The measurements are done in both fair-weather and thunderstorm conditions.

\section{Radio mechanism}

The main mechanism for radio emission from air showers is the deflection of electrons and positrons in the showers in opposite direction by the Lorentz force exerted by the geomagnetic field [4, 5]. They form a transverse current and thus emit radiation polarized linearly along the direction of the Lorentz force, i.e. $\hat{\mathbf{e}}_{\mathbf{v} \times \mathbf{B}}$, where $\mathbf{v}$ is the velocity of the shower and $\mathbf{B}$ is the geomagnetic field.

A secondary contribution is from charge excess which is due to a build-up of negative charge in the shower front [6, 7]. This also produces a radio pulse polarized radially with respect to the shower symmetry axis. The signal observed on the ground is the superposition of the two contributions and thus depends on the viewing angle.

Under thunderstorm conditions, strong atmospheric electric fields affect the motion of shower particles and thus influence the radio emission from the showers. The electric field can be decomposed into two components

$$
\mathbf{E}=\mathbf{E}_{\perp}+\mathbf{E}_{\|}
$$

where $\mathbf{E}_{\perp}$ and $\mathbf{E}_{\|}$are perpendicular and parallel to the shower axis, respectively. Due to $\mathbf{E}_{\perp}$, there is a net force acting on particles

$$
\mathbf{F}=\mathbf{q}\left(\mathbf{E}_{\perp}+\mathbf{v} \times \mathbf{B}\right) .
$$

which gives rise to a change in both magnitude and direction of the transverse current. Thus, the magnitude and polarization of the radiation from the tranverse current also change. Depending on the sign, the parallel component $\mathbf{E}_{\|}$may accelerate the particles and thus increase the number of particles at a certain energy. However, this effect is hardly seen in the signals observed by LOFAR LBA. These particles are trailing far behind the shower front, and therefore increase the intensity at lower frequencies [8]. Therefore, we assume that the parallel component vanishes in this work.

The complex voltages are expressed as $\varepsilon_{j}=E_{j}+i \hat{E}_{j}$ where $E_{j}$ is sample $j$ of the pulse and $\hat{E}_{j}$ is its Hilbert transform. The Stokes parameters are given by

$$
\begin{aligned}
I & =\frac{1}{n} \sum_{j=0}^{n-1}\left(|\varepsilon|_{j, \mathbf{v} \times \mathbf{B}}^{2}+|\varepsilon|_{j, \mathbf{v} \times \mathbf{v} \times \mathbf{B}}^{2}\right), \\
Q & =\frac{1}{n} \sum_{j=0}^{n-1}\left(|\varepsilon|_{j, \mathbf{v} \times \mathbf{B}}^{2}-|\varepsilon|_{j, \mathbf{v} \times \mathbf{v} \times \mathbf{B}}^{2}\right), \\
U+i V & =\frac{2}{n} \sum_{j=0}^{n-1}\left(\varepsilon_{j, \mathbf{v} \times \mathbf{B}} \varepsilon_{j, \mathbf{v} \times \mathbf{v} \times \mathbf{B}}^{*}\right),
\end{aligned}
$$

In the LOFAR analysis, the summations are calculated for $n=5$ samples of $5 \mathrm{~ns}$ each, centered around the peak of the pulse. Stokes I represents the intensity. The orientation of the linear polarization can be derived from Stokes Q and Stokes U. Stokes V is the amount of circular polarization. 

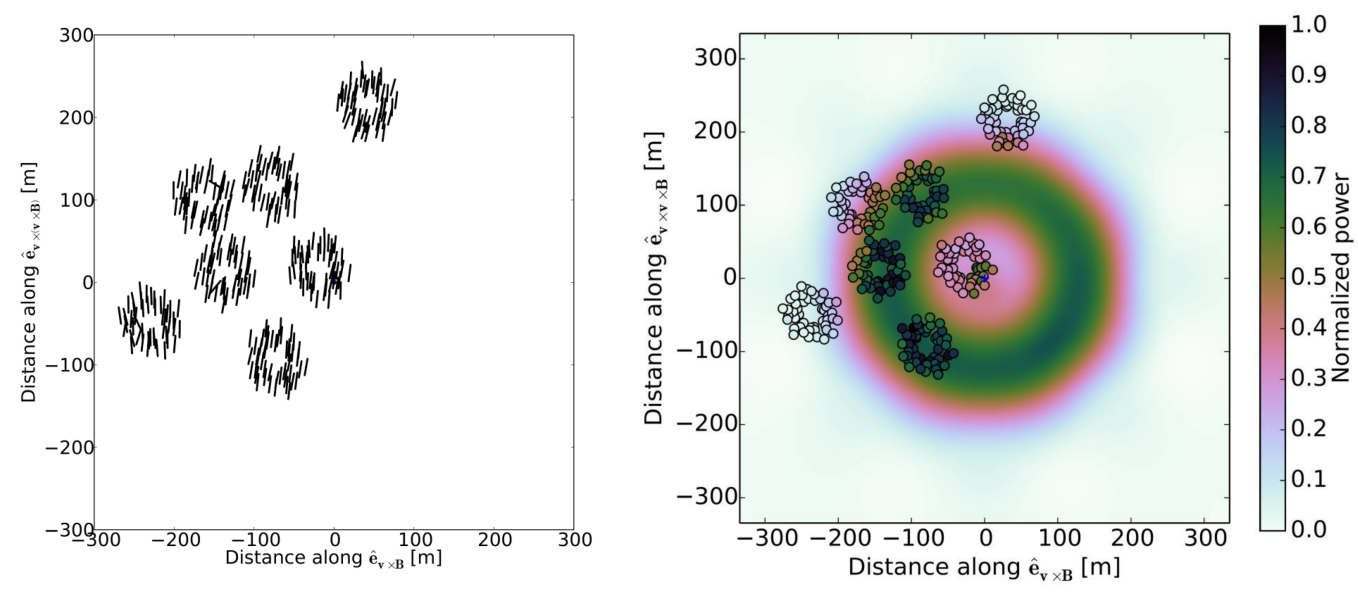

Figure 1. Left panel: Linear polarization footprint of an air shower under thunderstorm condition. Each line represents the orientation of the linear polarization for each antenna. Right panel: Intensity footprint of an air shower under thunderstorm condition. The background is the result from CoREAS simulation. Each small circle displays the intensity for each antenna. There is a good agreement between the data and the simulation.

\section{Fair-weather events vs thunderstorm events.}

As discussed above, the events observed under thunderstorm conditions show large differences shown in the intensity and polarizations compared to fair-weather events.

\subsection{Linear polarization}

The angle of linear polarization is given by $\psi=\frac{1}{2} \tan ^{-1}\left(\frac{U}{Q}\right)$. In fair-weather events, the polarization over all antennas is mainly along the expected $\hat{\mathbf{e}}_{\mathbf{v} \times \mathbf{B}}$ direction with some small deviation because of the contribution from charge excess. In thunderstorm events, the net force $\mathbf{F}$ changes the direction of the transverse current and thus the orientation of the linear polarization is no longer along the $\hat{\mathbf{e}}_{\mathbf{v} \times \mathbf{B}}$ direction, as it can be seen in the left panel of Fig. (1).

\subsection{Intensity footprint}

Unlike fair-weather events showing a bean-shaped intensity footprint due to the inteference of the transverse current and charge excess components, the ones in thunderstorm conditions show a ringlike structure as seen in the right panel of Fig. (1). The observed structure for this event can be understood as the effect of an electric field that is build up in two layers. The upper layer starts at a height of $8 \mathrm{~km}$ above the ground and extends to $2.9 \mathrm{~km}$ with a strength of $\left|E_{U}\right|=50 \mathrm{kV} / \mathrm{m}$. At the height of $2.9 \mathrm{~km}$, the electric field changes such that the net force is inversed and its strength reduces to $\left|E_{L}\right|=26.5 \mathrm{kV} / \mathrm{m}$. Two layers are needed to introduce the destructive interference between the emission from the two layers, which results in the ring-like structure in the intensity footprint.

\subsection{Circular polarization}

In fair-weather events, due to the time-shift between the pulses radiated from the transverse current component and the charge-excess component, there is a small amount of circular polarization. This 


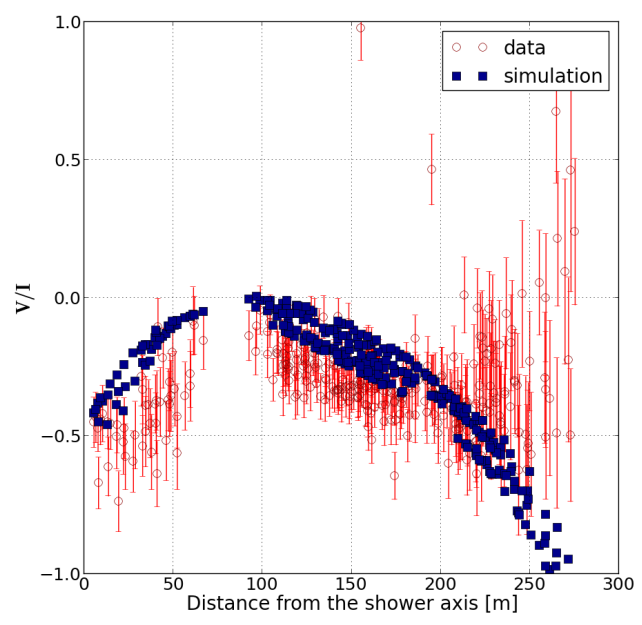

Figure 2. Circular polarization of an air shower under thunderstorm condition. The blue dots are simulated results and the red ones are data.

circular polarization vanishes at the core of the shower and depends on the azimuth angle of antennas [9]. However, in thunderstorm events we observe large amount of circular polarization near the core of the shower as shown in Fig. (2). This can only be explained by the change of the transverse current due to the change of the atmospheric electric field. Therefore, the full set of Stokes parameters helps to have a better understanding of the structure of atmospheric electric fields.

\section{Conclusion}

Using only the intensity footprint of the events measured under thunderstorm conditions, one can probe the atmospheric electric fields. It shows that we are sensitive to the height where the electric field changes and the relative strength between the fields in the two layers. The structure of electric fields can be recontructed better by using both the intensity and the polarization information.

\section{References}

[1] A. Dubinova et al., Phys. Rev. Lett. 115, 015002 (2015)

[2] J.R. Dwyer, M.A. Uman, Physics Reports 534, 147 (2014), the Physics of Lightning

[3] P. Schellart et al., Phys. Rev. Lett. 114, 165001 (2015)

[4] F.D. Kahn, I. Lerche, Royal Society of London Proceedings Series A 289, 206 (1966)

[5] O. Scholten, K. Werner, F. Rusydi, Astroparticle Physics 29, 94 (2008)

[6] G. Askaryan, J. Phys. Soc. Japan Vol: 17, Suppl. A-III (1962)

[7] K.D. de Vries et al., Astroparticle Physics 34, 267 (2010)

[8] T.N.G. Trinh et al., Phys. Rev. D 93, 023003 (2016)

[9] O. Scholten et al. (2016), accepted by Phys. Rev. D (11/2016) 